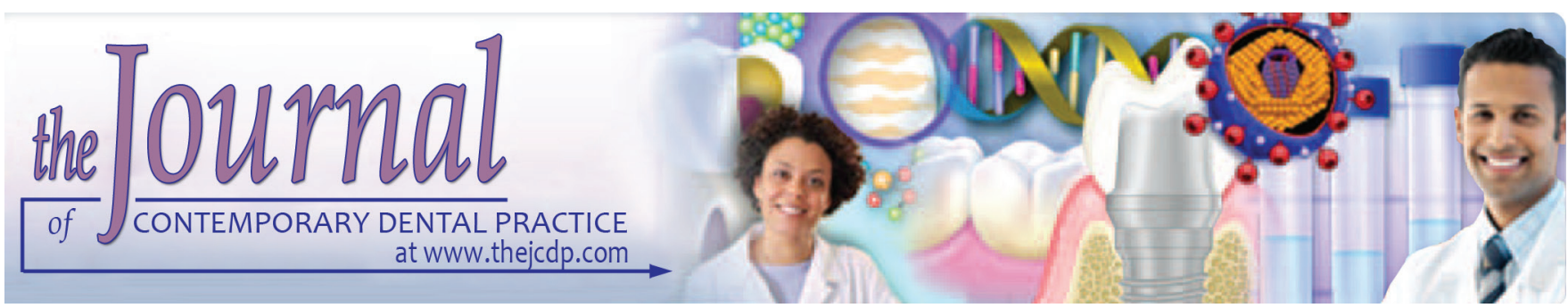

\title{
Ibuprofen and Low-level Laser Therapy for Pain Control during Fixed Orthodontic Therapy: A Systematic Review of Randomized Controlled Trials and Meta-analysis
}

${ }^{1}$ Faezeh Eslamipour, ${ }^{2}$ Saeed R Motamedian, ${ }^{3}$ Fahimeh Bagheri

\begin{abstract}
Introduction: To systematically review high-quality randomized controlled trials (RCTs) and meta-analysis on the effectiveness of use of ibuprofen and low-level laser therapy (LLLT) for pain control during fixed orthodontic appliance therapy.
\end{abstract}

Materials and methods: A web-based systematic search of PubMed and Medline database using relevant keywords was performed in August 2016 limited to the English language studies. Based on inclusion and exclusion criteria, RCTs utilizing blind approach were selected. The quality of studies was analyzed and only high-quality studies were included. Following data extraction, meta-analysis was performed by standardized mean difference Hedges' (adjusted) g with 95\% confidence interval.

Results: A total number of six studies (four ibuprofen and two LLLT) comprising 315 patients were included. Heterogeneity among ibuprofen studies was small, while large heterogeneity was found among LLLT studies. The results showed that both ibuprofen and LLLT could reduce pain intensity during fixed orthodontic therapy and during 17 days follow-up period. However, this reduction was statistically significant only at 6 to 24 hours postoperatively for ibuprofen and 2 hours and 3 to 7 days for LLLT $(p<0.05)$

Conclusion: Considering the limitations of the current metaanalysis, ibuprofen could alleviate orthodontic archwire activation pain during the 1st day with relatively high level of evidence. Low-level laser therapy could reduce this pain in the long term

\footnotetext{
${ }^{1}$ Dental Research Center, Department of Oral Public Health School of Dentistry, Isfahan University of Medical Sciences Isfahan, Islamic Republic of Iran

${ }^{2,3}$ Department of Orthodontics, Dental School, Shahid Behesht University of Medical Sciences, Tehran, Islamic Republic of Iran

Corresponding Author: Saeed R Motamedian, Department of Orthodontics, Dental School, Shahid Beheshti University of Medical Sciences, Tehran, Islamic Republic of Iran, Phone: +9130743676, e-mail: SR.Motamedian@yahoo.com
}

with limited evidence. Further well-designed RCTs are required to provide more evidence.

Keywords: Ibuprofen, Low-level laser therapy, Orthodontics, Pain, Randomized controlled trials, Review.

How to cite this article: Eslamipour F, Motamedian SR, Bagheri F. Ibuprofen and Low-level Laser Therapy for Pain Control during Fixed Orthodontic Therapy: A Systematic Review of Randomized Controlled Trials and Meta-analysis. J Contemp Dent Pract 2017;18(6):527-533.

Source of support: Nil

Conflict of interest: None

\section{INTRODUCTION}

Orthodontic pain is a common symptom that occurs 2 to 4 days following placement of fixed orthodontic appliances. ${ }^{1,2}$ Such discomfort is described as "tearing" in some patients and could hinder them to continue therapy. ${ }^{3}$ Some patients may stop toothbrushing due to the pain. Such pain increases during the 1st day and degrades after 1 week. ${ }^{2}$

The pain during orthodontic tooth movement is believed to be due to changes in periodontal compression, changes in blood flow, and resulted ischemia and inflammation. ${ }^{4}$ When periodontal tissues are compressed, inflammatory ligaments, such as histamine, bradykinin, prostaglandins, serotonin, and substance $\mathrm{P}$ are released and they start inflammatory reactions, resulting in patient pain. ${ }^{5}$

Factors influencing intensity of experienced pain include sex ${ }^{6,7}$ age, ${ }^{6,7}$ cultural differences, ${ }^{6,7}$ and state of emotions ${ }^{7}$ in addition to pain history. ${ }^{7}$ Some authors have questioned the role of sex on pain intensity and suggested that such difference is more likely due to cultural differences rather than physiological reasons. ${ }^{6,8}$

The application of nonsteroidal anti-inflammatory drugs (NSAIDs) is one of the preferred methods for pain 
control during fixed orthodontic therapy. They could inhibit cyclooxygenase and prevent prostaglandins biosynthesis from arachidonic acid. ${ }^{9}$ The first report on use of ibuprofen for orthodontic tooth movement pain relieve by Ngan et a ${ }^{10}$ showed the analgesic efficacy of ibuprofen compared to placebo and aspirin. The NSAIDs could be used both pre- and postoperatively.

On the contrary, low-level laser therapy (LLLT) has been increasingly applied in dentistry due to its regenerative, analgesic, and anti-inflammatory features as well as enamel conditioning. ${ }^{11-13}$ Its low output energy does not cause temperature rise above $36.5^{\circ} \mathrm{C}$. Mechanism of LLLT pain control has not been explained, yet. Some suggested that the effect of LLLT on neuronal and inflammatory cells is responsible for pain relief. ${ }^{14}$ Although the positive effect of LLLT on pain reduction during fixed orthodontic therapy has been demonstrated in some studies, ${ }^{3,13}$ Lim et $\mathrm{al}^{15}$ found no significant difference in pain following application of LLLT.

Systematic reviews on randomized controlled trials (RCTs) could determine the level of evidence for use of interventions in human. Previous literature reviews demonstrated the effectiveness of analgesic drugs in pain reduction during fixed orthodontic therapy ${ }^{16}$ while on the effectiveness of LLLT, the evidence is limited. ${ }^{13}$ The aim of this study was to systematically review high-quality RCTs and meta-analysis on the effectiveness of use of ibuprofen and LLLT for pain control during fixed orthodontic therapy.

\section{MATERIALS AND METHODS}

\section{Study Design}

In this review, RCTs evaluating the effectiveness of use of single-dose ibuprofen or LLLT in pain management of fixed orthodontic therapy in humans were included. Only studies which used visual analog system (VAS) for assessment of pain degree were included and other methods were excluded. Moreover, studies that did not mention when the pain intensity was assessed (during chewing) were excluded. Studies on fixed orthodontic therapy were included and craniofacial syndromes, orthosurgery, removable orthodontic therapy, and elastic separator placement were excluded. In addition, other methods for pain control apart from ibuprofen and LLLT were excluded. The drug or laser administration protocol varied among studies, and therefore, it was not considered as an inclusion/exclusion criteria.

\section{Electronic Search and Study Selection}

An electronic search was performed using PubMed and Medline until August 2016 limited to English language and human studies. A combination of relevant keywords was used according to PICO: Problem/Patient/
Population Intervention/Indicator Comparison Outcome of Interest:

Patient:

“Orthodontics"[Mesh] OR orthodontic*

AND Intervention and Control:

"Anti-Inflammatory Agents, Non-Steroidal"[Mesh] OR NSAID* OR "Ibuprofen"[Mesh] OR "Laser Therapy"[Mesh] OR "Lasers"[Mesh] OR "Low-Level Light Therapy"[Mesh]

AND Control:

"Randomized Controlled Trial" [Publication Type] OR random* OR control* OR Placebos"[Mesh]

AND Outcome:

"Pain Measurement"[Mesh] OR "Pain"[Mesh] OR "Visual Analog Scale"[Mesh]

In addition, the references of the included studies were searched for further relevant studies. Initial screening of titles and abstracts was carried out and full texts of the potentially eligible studies were obtained for further evaluation. Studies were included based on established inclusion/exclusion criteria by two reviewers separately. Disagreements were discussed with the third reviewer.

\section{Quality Assessment}

To reduce meta-analysis bias, two reviewers assessed the quality of included studies based on Jadad et a ${ }^{17}$ and only studies with low risk of bias (score $\geq 2$ ) were included. Jadad criteria (maximum 5 scores) include randomization (1 score), appropriate method of randomization (1 score), blinding (1 score), appropriate method of blinding (1 score), and handling dropouts (1 score). ${ }^{17}$

\section{Data Extraction and Meta-analysis}

Relevant data including number of patients, mean age, and mean VAS of each group (test and control), as well as drug or laser administration protocol,were extracted from each study.

Meta-analysis was carried out by Stata 13 (College Station, TX, USA). For every pair-wise comparison standardized mean difference (SMD) Hedges' (adjusted) g with confidence interval $95 \%$ was obtained. Heterogeneity between studies was measured by $\mathrm{I}^{2}$ which ranges between 0 and $100 \%$. For values more than $50 \%$ showing large heterogeneity ${ }^{18}$ random effects model was used. If $\mathrm{I}^{2}$ was $<50 \%$, fixed effects model was applied. Cochran's Q was also calculated, but only its significance was considered. The level of significance was set at 0.05 .

This study was performed in compliance with the preferred reporting items for systematic reviews and meta-analyses statement. ${ }^{19}$

\section{RESULTS}

The electronic literature search identified 92 studies (Graph 1), of which full-text of 28 studies were assessed 


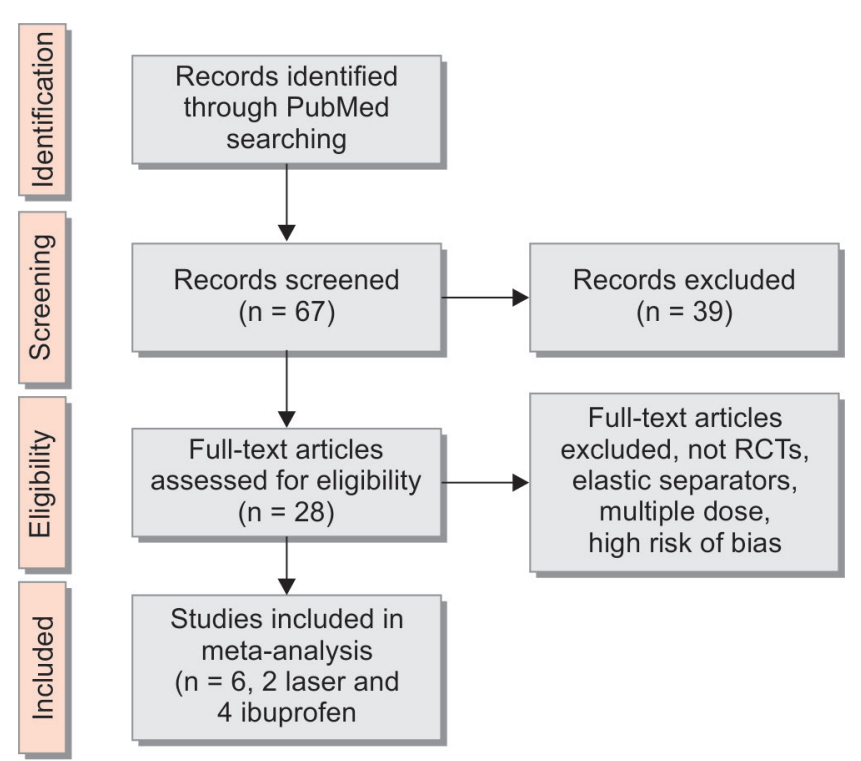

Graph 1: Review flow diagram

for eligibility. No further publication was provided by the hand search. A total of six studies met the inclusion criteria and were included in the meta-analysis (Table 1). The included studies comprised a total number of 315 patients (157 received interventions and 158 received placebos).

Low-level Laser Therapy vs Placebo

A total number of two studies comprising 196 patients compared the analgesic effect of LLLT vs placebo. The SMD at different time points within 7 days after activation of fixed orthodontic treatment was in favor of LLLT (Table 2). As apparent in forest plot (Graph 2A-F), however, the analgesic effect of LLLT compared to placebo reached significant level only at 2 hours and 3 and 7 days, showing that LLLT could control pain mostly in long term. The SMD was -8.094 (95\% confidence interval $[\mathrm{CI}]=-9.213$ to $-6.975, \mathrm{p}<0.001),-6.126(95 \%$ $\mathrm{CI}=-14.921$ to $1.373, \mathrm{p}<0.001)$, and $-2.846(95 \% \mathrm{CI}=$ -3.363 to $-2.329, p<0.001)$ at hours and 3 and 7 days respectively. Heterogeneity among studies was high $\left(\mathrm{I}^{2}>99 \%\right.$ when both studies were included).

\section{Ibuprofen vs Placebo}

One hundred and nineteen patients were enrolled in four studies comparing pain relief effect of ibuprofen vs placebo. The SMD at different time points within 7 days after activation of fixed orthodontic treatment was in favor of ibuprofen (Table 3). Forest plot shows that ibuprofen is significantly more effective for pain management compared to placebo from 6 hours after until 24 hours (Graph 3A-G). The SMD was -0.450 (95\% CI = -0.817 to $-0.083, \mathrm{p}=0.016),-0.629(95 \% \mathrm{CI}=-1.086$ to $-0.173, \mathrm{p}=0.007)$, and $-0.433(95 \% \mathrm{CI}=-0.804$ to -0.063 , $\mathrm{p}=0.022$ ) at 6 hours, night, and 24 hours after activation of fixed orthodontic treatment respectively. Heterogeneity of included studies was $<50 \%$.

\section{DISCUSSION}

Since tooth movement could cause pain and discomfort, attempts have been performed to alleviate such pain and increase patient satisfaction. Previously, several reviews have analyzed the results of the clinical trials to find the most efficient method of fixed orthodontic treatment pain management. ${ }^{13,16,24-27}$ However, they mostly did not consider several variables which could influence the results and cause bias. The number of included studies in this review was fewer compared to previous reviews as the aim of this review was to combine the results

Table 1: Data summary of six qualified trials and their quality assessment based on the Jadad score ${ }^{17}$

\begin{tabular}{llllll}
\hline Study & $\begin{array}{l}\text { Number of } \\
\text { treatment }\end{array}$ & $\begin{array}{l}\text { Number of } \\
\text { placebo }\end{array}$ & Intervention & Protocol & $\begin{array}{l}\text { Jadad } \\
\text { score }\end{array}$ \\
\hline Domínguez A, Velásquez $^{20}$ & 60 & 60 & LLLT & $830 \mathrm{~nm}, 100 \mathrm{~mW}, 22$ seconds, $80 \mathrm{~J} / \mathrm{cm}^{2}$ & 2 \\
Turhani et al $^{3}$ & 38 & 38 & LLLT & $670 \mathrm{~nm}, 75 \mathrm{~mW}, 30$ seconds, $140 \mathrm{~mW} / \mathrm{cm}^{2}$ & 2 \\
Polat and Karaman $^{21}$ & 20 & 20 & lbuprofen & $600 \mathrm{mg}$ & 2 \\
Polat et al $^{5}$ & 20 & 20 & lbuprofen & $400 \mathrm{mg}$ & 3 \\
Salmassian et al $^{22}$ & 19 & 20 & lbuprofen & $400 \mathrm{mg}$ & 4 \\
Farzanegan et al $^{23}$ & 10 & 10 & lbuprofen & $400 \mathrm{mg}$ & 3 \\
\hline
\end{tabular}

Table 2: Meta-analysis data summary: Low-level laser therapy vs placebo

\begin{tabular}{|c|c|c|c|c|c|}
\hline \multirow[b]{2}{*}{ Time (hours) } & \multirow[b]{2}{*}{$S M D$} & \multirow[b]{2}{*}{$95 \% \mathrm{Cl}$} & \multicolumn{2}{|c|}{ Heterogeneity } & \multirow[b]{2}{*}{$p$-value } \\
\hline & & & $P^{2}(\%)$ & $p$-value & \\
\hline 2 & -8.094 & -9.213 to -6.975 & 0 & 0.586 & $<0.001$ \\
\hline 6 & -6.133 & -14.228 to 1.961 & 99.1 & $<0.001$ & 0.138 \\
\hline 24 & -6.774 & -14.921 to 1.373 & 99.0 & $<0.001$ & 0.103 \\
\hline 48 & -9.619 & -21.675 to 2.437 & 99.1 & $<0.001$ & 0.118 \\
\hline 72 & -6.126 & -7.004 to -5.248 & 0 & 0.940 & $<0.001$ \\
\hline 7 days & -2.846 & -3.363 to -2.329 & 0 & 0.622 & $<0.001$ \\
\hline
\end{tabular}




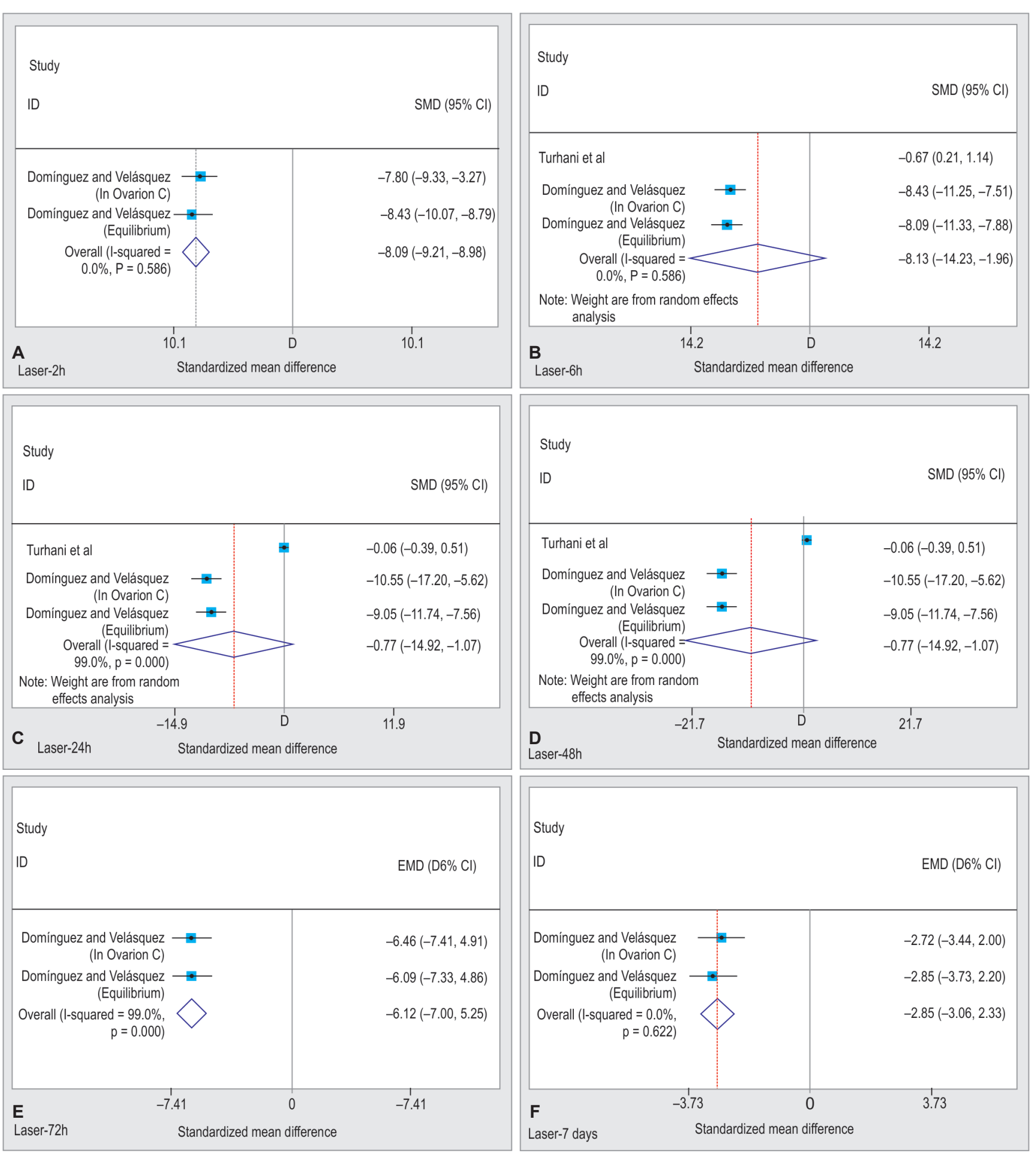

Graph 2A to F: Result of meta-analysis for LLLT and placebo, reported in standard mean difference (95\% confidence interval) at different time points: (A) 2 hours; (B) 6 hours; (C) 24 hours; (D) 48 hours; (E) 72 hours; and (F) 7 days. Forest plot shows analgesic effect of LLLT compared with placebo at 2 hours and 3 and 7 days after activation of fixed orthodontic treatment

Table 3: Meta-analysis data summary: Ibuprofen vs placebo

\begin{tabular}{llllll}
\hline & \multicolumn{2}{l}{$\begin{array}{l}\text { Standardized mean } \\
\text { difference }\end{array}$} & $95 \% \mathrm{Cl}$ & \multicolumn{2}{c}{ Heterogeneity } \\
\cline { 3 - 5 } Time (hours) & -0.056 & -0.419 to 0.306 & 19.4 & 0.293 & 0.760 \\
6 & -0.450 & -0.817 to -0.083 & 0 & 0.445 & 0.016 \\
At night & -0.629 & -1.086 to -0.173 & 31.1 & 0.234 & 0.007 \\
24 & -0.433 & -0.804 to -0.063 & 46.9 & 0.130 & 0.022 \\
48 & -0.200 & -0.564 to 0.164 & 18.2 & 0.300 & 0.281 \\
72 & -0.076 & -0.439 to 0.288 & 33.9 & 0.209 & 0.684 \\
7 days & -0.048 & -0.411 to 0.316 & 27.4 & 0.248 & 0.797 \\
\hline
\end{tabular}




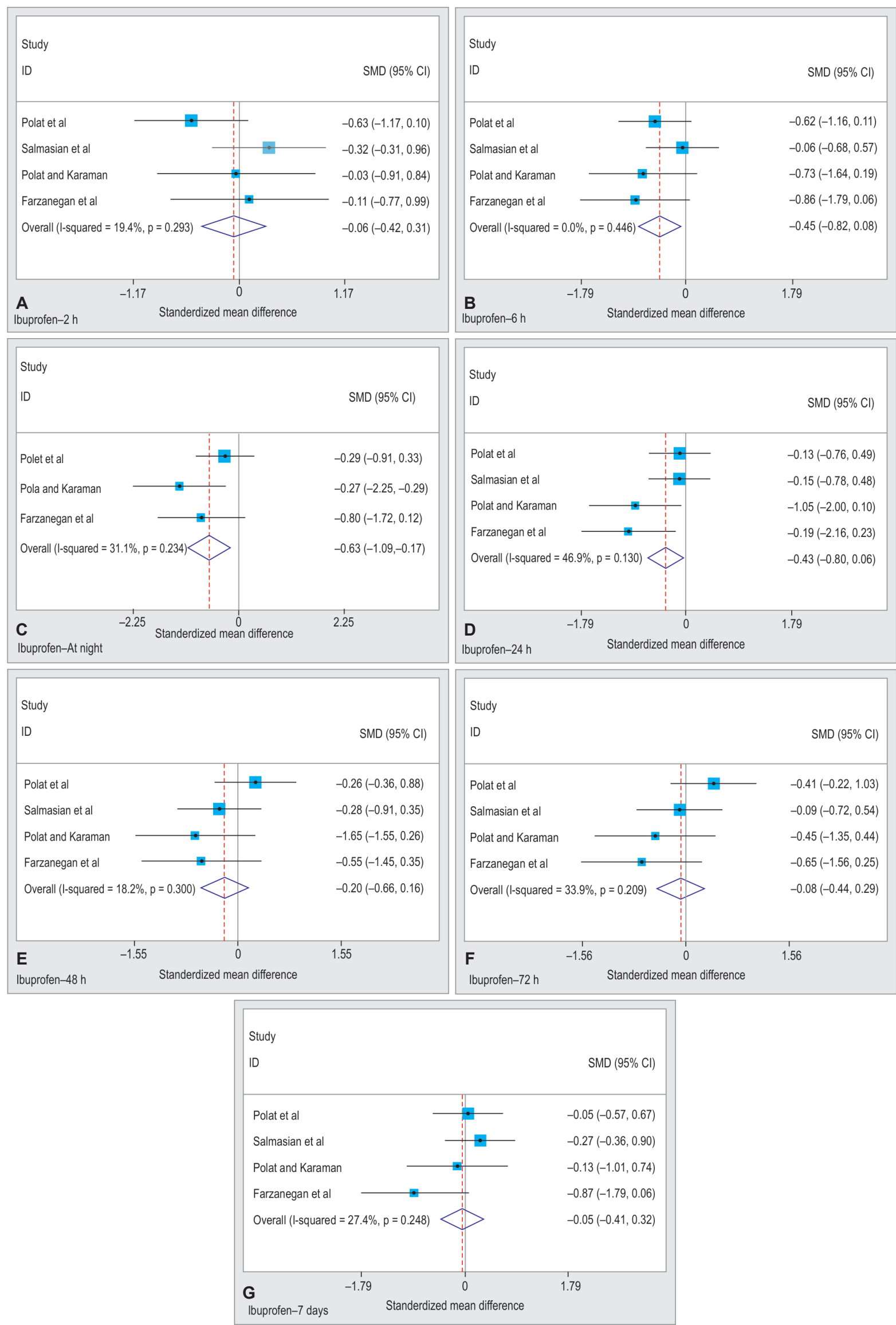

Graph 3A to G: Result of meta-analysis for ibuprofen and placebo reported in standard mean difference (95\% confidence interval) at different time points: (A) 2 hours; (B) 6 hours; (C) night; (D) 24 hours; (E) 48 hours; (F) 72 hours; and (G) 7 days. Forest plot shows analgesic effect of ibuprofen compared to placebo from at 2 hours until 24 hours after activation of fixed orthodontic treatment 
of studies with homogenous method. In the review of Xiaoting et $\mathrm{al}^{16}{ }^{16}$ they included the study of Tortamano et $\mathrm{al}^{28}$ for LLLT in which the authors assessed pain by a survey. He et $\mathrm{al}^{13}$ and Ren et $\mathrm{al}^{25}$ included all studies on LLLT not considering the frequency of laser treatment. This review only included high-quality studies which assessed pain alleviating effect of one-time application of laser with VAS.

In addition, several studies, such as Ngan et al, ${ }^{10}$ Steen Law et al, ${ }^{29}$ Bradley et $a{ }^{30}$ and Minor et $\mathrm{al}^{31}$ who measured analgesic effect of ibuprofen following elastic separator insertion were included in previous meta-analyses, ${ }^{16,24,26,27}$ while they were excluded from this review as the pain might vary with pain caused by archwire placement.

The results of this meta-analysis showed that ibuprofen could reduce pain intensity during the 1st day after activation of archwire. The level of evidence was high and included studies were homogenous. Previously, Angelopoulou et $\mathrm{al}^{24}$ and Xiaoting et $\mathrm{ll}^{16}$ revealed analgesic effect of ibuprofen 2 to 6 hours and 6 hours to 3 days after archwire activation or separator insertion.

The two included studies on the effect of LLLT were heterogonous; hence, the risk of bias is high. The results showed that LLLT could alleviate pain after 2 hours, 3 and 7 days. Although it is believed that LLLT is a noninvasive method with no adverse effect for pain management, the long-time needed (32-37.5 minutes) for application to both dental arches prohibit its routine usage. ${ }^{16}$

One of the limitations of this study was the limited amount of comparative data. Hence, it seems that highquality RCTs should further examine the effectiveness of pain management methods during fixed orthodontic treatment.

\section{CONCLUSION}

Ibuprofen can lessen pain at 6 hours after orthodontic archwire activation, while its effect is only statistically significant until 24 hours. The level of the evidence was relatively high.

Low-level laser therapy also could reduce pain after 3 to 7 days following archwire activation. However, the studies were heterogeneous.

\section{REFERENCES}

1. Jones ML. An investigation into the initial discomfort caused by placement of an archwire. Eur J Orthod 1984 Feb;6(1):48-54.

2. Ngan P, Kess B, Wilson S. Perception of discomfort by patients undergoing orthodontic treatment. Am J Orthod Dentofacial Orthop 1989 Jul;96(1):47-53.

3. Turhani D, Scheriau M, Kapral D, Benesch T, Jonke E, Bantleon HP. Pain relief by single low-level laser irradiation in orthodontic patients undergoing fixed appliance therapy. Am J Orthod Dentofacial Orthop 2006 Sep;130(3):371-377.
4. Furstman L, Bernick S. Clinical considerations of the periodontium. Am J Orthod 1972 Feb;61(2):138-155.

5. Polat O, Karaman AI, Durmus E. Effects of preoperative ibuprofen and naproxen sodium on orthodontic pain. Angle Orthod 2005 Sep;75(5):791-796.

6. Bergius M, Kiliaridis S, Berggren U. Pain in orthodontics. A review and discussion of the literature. J Orofac Orthop 2000 Mar;61(2):125-137.

7. Andreasen JO, Peterson J, Laskin DM. Textbook and color atlas of tooth impactions. Am J Orthod Dentofac Orthop 1997 Sep;112(3):354.

8. Bergius M, Berggren U, Kiliaridis S. Experience of pain during an orthodontic procedure. Eur J Oral Sci 2002 Apr;110(2): 92-98.

9. Kehoe MJ, Cohen SM, Zarrinnia K, Cowan A. The effect of acetaminophen, ibuprofen, and misoprostol on prostaglandin E2 synthesis and the degree and rate of orthodontic tooth movement. Angle Orthod 1996 Oct;66(5):339-349.

10. Ngan P, Wilson S, Shanfeld J, Amini H. The effect of ibuprofen on the level of discomfort in patients undergoing orthodontic treatment. Am J Orthod Dentofacial Orthop 1994 Jul;106(1):88-95.

11. Silva Júnior AN, Pinheiro AL, Oliveira MG, Weismann $R$, Ramalho LM, Nicolau RA. Computerized morphometric assessment of the effect of low-level laser therapy on bone repair: an experimental animal study. J Clin Laser Med Surg 2002 Apr;20(2):83-87.

12. Roberts-Harry DP. Laser etching of teeth for orthodontic bracket placement: a preliminary clinical study. Lasers Surg Med 1992;12(5):467-470.

13. He WL, Li CJ, Liu ZP, Sun JF, Hu ZA, Yin X, Zou SJ. Efficacy of low-level laser therapy in the management of orthodontic pain: a systematic review and meta-analysis. Lasers Med Sci 2013 Nov;28(6):1581-1589.

14. Bjordal JM, Johnson MI,Iversen V,AimbireF, Lopes-Martins RA. Low-level laser therapy in acute pain: a systematic review of possible mechanisms of action and clinical effects in randomized placebo-controlled trials. Photomed Laser Surg 2006 Apr;24(2):158-168.

15. Lim HM, Lew KK, Tay DK. A clinical investigation of the efficacy of low level laser therapy in reducing orthodontic postadjustment pain. Am J Orthod Dentofacial Orthop 1995 Dec;108(6):614-622.

16. Xiaoting L, Yin T, Yangxi C. Interventions for pain during fixed orthodontic appliance therapy. A systematic review. Angle Orthod 2010 Sep;80(5):925-932.

17. Jadad AR, Moore RA, Carroll D, Jenkinson C, Reynolds DJ, Gavaghan DJ, McQuay HJ. Assessing the quality of reports of randomized clinical trials: is blinding necessary? Control Clin Trials 1996 Feb;17(1):1-12.

18. Huedo-Medina TB, Sánchez-Meca J, Marín-Martínez F, Botella J. Assessing heterogeneity in meta-analysis: $Q$ statistic or $\mathrm{I}^{2}$ index? Psychol Methods 2006 Feb;11(2):193-206.

19. Liberati A, Altman DG, Tetzlaff J, Mulrow C, Gøtzsche PC, Ioannidis JP, Clarke M, Devereaux PJ, Kleijnen J, Moher D, et al. The PRISMA statement for reporting systematic reviews and meta-analyses of studies that evaluate health care interventions: explanation and elaboration. Ann Intern Med 2009 Aug;151(4):W65-W94.

20. Domínguez A, Velásquez SA. Effect of low-level laser therapy on pain following activation of orthodontic final archwires: 
a randomized controlled clinical trial. Photomed Laser Surg 2013 Jan;31(1):36-40.

21. Polat O, Karaman AI. Pain control during fixed orthodontic appliance therapy. Angle Orthod 2005 Mar;75(1):214-219.

22. Salmassian R, Oesterle LJ, Shellhart WC, Newman SM. Comparison of the efficacy of ibuprofen and acetaminophen in controlling pain after orthodontic tooth movement. Am J Orthod Dentofacial Orthop 2009 Apr;135(4):516-521.

23. Farzanegan F, Zebarjad SM, Alizadeh S, Ahrari F. Pain reduction after initial archwire placement in orthodontic patients: a randomized clinical trial. Am J Orthod Dentofacial Orthop 2012 Feb;141(2):169-173.

24. Angelopoulou MV, Vlachou V,Halazonetis DJ. Pharmacological management of pain during orthodontic treatment: a metaanalysis. Orthod Craniofac Res 2012 Apr;15(2):71-83.

25. Ren C, McGrath C, Yang Y. The effectiveness of low-level diode laser therapy on orthodontic pain management: a systematic review and meta-analysis. Lasers Med Sci 2015 Sep;30(7):1881-1893.

26. Li FJ, Zhang JY, Zeng XT, Guo Y. Low-level laser therapy for orthodontic pain: a systematic review. Lasers Med Sci 2015 Aug;30(6):1789-1803.
27. Sandhu SS, Cheema MS, Khehra HS. Comparative effectiveness of pharmacologic and nonpharmacologic interventions for orthodontic pain relief at peak pain intensity: a Bayesian network meta-analysis. Am J Orthod Dentofacial Orthop 2016 Jul;150(1):13-32.

28. Tortamano A, Lenzi DC, Haddad AC, Bottino MC, Dominguez GC, Vigorito JW. Low-level laser therapy for pain caused by placement of the first orthodontic archwire: a randomized clinical trial. Am J Orthod Dentofacial Orthop 2009 Nov;136(5):662-667.

29. Steen Law SL, Southard KA, Law AS, Logan HL, Jakobsen JR. An evaluation of preoperative ibuprofen for treatment of pain associated with orthodontic separator placement. Am J Orthod Dentofacial Orthop 2000 Dec;118(6):629-635.

30. Bradley RL, Ellis PE, Thomas P, Bellis H, Ireland AJ, Sandy JR. A randomized clinical trial comparing the efficacy of ibuprofen and paracetamol in the control of orthodontic pain. Am J Orthod Dentofacial Orthop 2007 Oct;132(4):511-517.

31. Minor V, Marris CK, McGorray SP, Yezierski R, Fillingim R, Logan H, Wheeler TT. Effects of preoperative ibuprofen on pain after separator placement. Am J Orthod Dentofacial Orthop 2009 Oct;136(4):510-517. 